\section{ORIGINAL RESEARCH}

\author{
C.B. Nauer \\ A. Eichenberger \\ P. Dubach \\ J. Gralla \\ M. Caversaccio
}

\title{
CT Radiation Dose for Computer-Assisted Endoscopic Sinus Surgery: Dose Survey and Determination of Dose-Reduction Limits
}

BACKGROUND AND PURPOSE: Computer-assisted navigation is increasingly used in functional endoscopic sinus surgery (FESS) to prevent injury to vital structures, necessitating preparative CT and, thus, radiation exposure. The purpose of our study was to investigate currently used radiation doses for CT in computer-assisted navigation in sinus surgery (CAS-CT) and to assess minimal doses required.

MATERIALS AND METHODS: A questionnaire inquiring about dose parameters used for CAS-CT was sent to 30 radiologic institutions. The feasibility of low-dose registration was tested with a phantom. The influence of CAS-CT dose on technical accuracy and on the practical performance of 5 ear, nose, and throat (ENT) surgeons was evaluated with cadaver heads.

RESULTS: The questionnaire response rate was $63 \%$. Variation between minimal and maximal dose used for CAS-CT was 18-fold. Phantom registration was possible with doses as low as $1.1 \mathrm{mGy}$. No dose dependence on technical accuracy was found. ENT surgeons were able to identify anatomic landmarks on scans with a dose as low as $3.1 \mathrm{mGy}$.

CONCLUSIONS: The vast dose difference between institutions mirrors different attitudes toward image quality and radiation-protection issues rather than being technically founded, and many patients undergo CAS-CT at higher doses than necessary. The only limit for dose reduction in CT for computerassisted endoscopic sinus surgery is the ENT surgeon's ability to cope with impaired image quality, whereas there is no technically justified lower dose limit. We recommend, generally, doses used for the typical diagnostic low-dose sinus CT (120 kV/20-50 mAs). When no diagnostic image quality is needed, even a reduction down to a third is possible.

mage-guided navigation systems are increasingly used for minimally invasive techniques like functional endoscopic surgery of the paranasal sinuses (FESS) ${ }^{1,2}$ and other ear, nose, and throat (ENT) surgical procedures ${ }^{3-5}$ or in neurosurgery. ${ }^{6}$ In FESS, only a limited view of the anatomy is warranted, and the use of these systems aims at avoiding injury to important anatomic structures around the orbit and the anterior skull base, especially the internal carotid artery, the optic nerve, and the floor of the anterior skull base..$^{5}$ Although these systems improve orientation in narrow anatomic compartments, they lead to an increased intervention time ${ }^{3}$ and the need for preoperative imaging. Although endoscopic sinus surgery with intraoperative MR imaging guidance is feasible, ${ }^{7}$ the use of CT-based datasets for navigation is still standard, involving radiation exposure to the patient. This is of importance because many young patients are treated surgically for hyperplastic rhinosinusitis, and for younger people, radiation-induced cancer risk is considerably higher. ${ }^{8}$ It is, therefore,

Received July 31, 2008; accepted after revision September 19.

From the Institute for Diagnostic and Interventional Neuroradiology (C.B.N., J.G.) and the Department of Otorhinolaryngology/Head and Neck Surgery (A.E., P.D., M.C.), University Hospital and University of Berne, Berne, Switzerland.

Marco Caversaccio is supported by the Swiss National Research Foundation for the project Computer-Aided Surgery around the Head in Computer Aided and Image Guided Medical Intervention.

Please address correspondence to Claude Nauer, MD, Institute for Diagnostic and Interventional Neuroradiology, Inselspital/University Hospital, Freiburgstr 4, CH-3010 Berne, Switzerland; e-mail: claude.nauer@insel.ch; and Marco Caversaccio, Department of Otorhinolaryngology/Head and Neck Surgery, Inselspital/University Hospital, Freiburgstr 4, CH-3010 Berne, Switzerland; e-mail: marco.caversaccio@insel.ch

Indicates open access to non-subscribers at www.ajnr.org

DOI 10.3174/ajnr.A1378 mandatory to perform computer-assisted navigation in sinus surgery (CAS-CT) with radiation doses as low as possible.

At the start of our study, 3 protocols for sinus CT imaging were used at our neuroradiologic department. First, we used a dedicated low-dose protocol for sinusitis (sinusitis CT, 30 $\mathrm{mAs}$ ) as amply described in the literature. ${ }^{9-11}$ Second, we used a high-dose protocol for optimal soft-tissue delineation (140 $\mathrm{mAs}$ ). Third, we had a dedicated protocol for CAS-CT (210 $\mathrm{mAs}$ ). While checking all CT protocols at our neuroradiologic department for radiation-reduction potential, we considered lowering the dose for CAS-CT. Consultation with the manufacturer gave no evidence that technical reasons inherent in the image-guidance system justify a higher dose for CAS-CT, but we did not find dedicated low-dose protocols for CAS-CT in the literature either. Indeed, there are a few potential problems conceivable with low-dose imaging for navigation.

First, the CT dataset must be matched to the patient's facial features; this is the so-called registration. Image noise in lowdose scans might interfere with the registration algorithm, especially because a surface-rendered image (Fig 1) is used for registration. Furthermore, navigation accuracy could deteriorate with growing noise. This might prove disastrous in the vicinity of vital structures. Last, prominence of anatomic structures needed for intraoperative orientation by ENT surgeons could suffer.

To the best of our knowledge, there has been neither a survey on radiation doses from CAS-CT nor a systematic experimental evaluation of minimal doses required for multidetector CT in computer-assisted sinus surgery until now. The aim of our study was to look into currently used radiation doses for CAS-CT (What do others do?), to define a clinically 

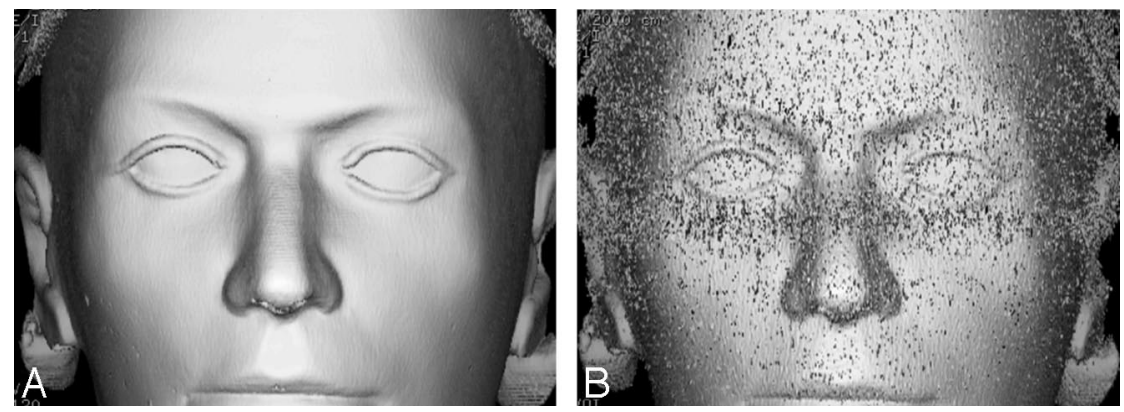

Fig 1. $A$ and $B$, A surface-rendered image reconstructed on an Advantage Windows workstation (GE Healthcare) from a CT dataset performed with a CTDI ${ }_{\text {vol }}$ of $65 \mathrm{mGy}(A)$ shows no discernible noise, whereas with a CTDI ${ }_{\text {vol }}$ of $9.5 \mathrm{mGy}(B)$, delineation of facial contours is impaired due to image noise which might, according to our initial hypothesis, render surface registration impossible.

\begin{tabular}{|c|c|c|}
\hline $\mathrm{CTDI}_{\text {vol }}$ (mGy) & $\mathrm{kV}$ & $\mathrm{mAs}$ \\
\hline 65 & 120 & 210 \\
\hline 9.5 & 120 & 30 \\
\hline 6.3 & 120 & 20 \\
\hline & 100 & 20 \\
\hline 3.1 & 120 & 10 \\
\hline & 100 & 10 \\
\hline 1.1 & 80 & 10 \\
\hline
\end{tabular}

Note:-CTDI ${ }_{\text {vol }}$ indicates volumetric CT-dose index.

applicable low-dose protocol for our institution, and to investigate the lowest dose thresholds for safe navigation.

\section{Materials and Methods}

No consent from the local ethics committee was required for this experimental study.

\section{Dose Survey}

A questionnaire was sent to 30 radiology departments, among them university hospitals, primary care hospitals, and private radiology CT practices. We included only institutions that were supposed to use CT-based navigation tools. Information on model and manufacturer of the CT scanner and the navigation system and dose-relevant scanning parameters (tube voltage, tube current-time product, and volumetric CT-dose index $\left[\mathrm{CTDI}_{\mathrm{vol}}\right]$ ) for CAS-CT and diagnostic sinusitis CT was requested.

\section{Evaluation of Registration Feasibility and Dose Dependence of Navigation Accuracy}

To investigate potential problems associated with low-dose scanning and to define the lowest possible dose for registration, navigation, and FESS, we conducted phantom and cadaver head experiments. For CAS-CT, overlapping $1.25-\mathrm{mm}$ sections with a FOV of $20 \mathrm{~cm}$ were acquired with an 8-section scanner (LightSpeed Ultra; GE Healthcare, Milwaukee, Wis). Images were reconstructed in edge-enhancing algorithms. The face from the upper edge of the frontal sinus to the upper tooth row, including the tip of the nose, was imaged to allow reliable registration. To assess registration feasibility at very low doses, we scanned a tissue-equivalent head phantom (XR-100; Alderson Research, Stamford, Conn) with low-dose scanning (Table), and registration was performed with each dataset. In a second step, dose dependence of navigation accuracy was evaluated with 4 human cadaver heads. As a reference dose, the initially used high dose $(120 \mathrm{kV} / 210$ mAs) was also included.

When preparing computer-assisted endoscopic navigation, a correlation between the dataset and the patient's facial contours (ie, registration process) must be performed first. For this purpose, the sys- tem calculates a 3D surface-rendered model from the image data. Correlation is then achieved touching firmly attached markers, such as bone-anchored screws or fiducials glued onto the skin, with a pointer, which is tagged with reflection markers (see the paragraph on the optical link below). Alternatively, multiple discrete surface landmarks can be defined by touching the face with a pointer or setting laser light points (surface registration). Screw-based registration has proved to be the most accurate method ${ }^{4}$ and was, therefore, chosen for our study to keep influential factors, other than dose-associated image quality, as limited as possible.

Correlation between the patient's position and the instruments is maintained during the intervention by an optical, electromechanical, electromagnetic, or sonographic link. ${ }^{12}$ During the intervention, the CT data, including multiplanar reformations in coronal and sagittal orientations, are displayed by the navigation system (Fig 2). At our institution, an optically guided system (VectorVision; BrainLAB, Feldkirchen, Germany) is in service. Optically guided systems use infrared light, which is reflected by markers attached to the patient's head and the instruments, allowing determination of the instrument position in relation to patient anatomy.

For the evaluation of navigation accuracy, reference points, which can be identified on the CT scan and on the cadaver specimen, were defined. For this purpose, small screws were drilled into the bone in the region of the frontozygomatic suture (screws 1 and 2), the nasion (screw 3), the nasal spine (screw 4), the pterygoid process (screw 5), and the sphenoid (screw 6). Screws 5 and 6 were placed endoscopically. Additionally, the screw holding the reflection marker in place on the forefront was included (screw 7). The screw heads had a central pit where the pointer used for accuracy measurement could be placed jiggle-free. These points were marked with the software iPlan (BrainLab, Feldkirchen, Germany) (Fig 3) at adequate magnification. When the pointer tip is put exactly at the marked location, accuracy is calculated as the root mean square error (RSME) by the navigation system (BrainLab). ${ }^{1}$ This value indicates the maximal deviation between patient and image data. ${ }^{4}$ RMSE was recorded after placing the pointer tip in the screw head pit in line with the main axis of the screw. Screws 1-4 were used as bone-anchored fiducials for registration.

\section{Statistical Analysis}

For descriptive statistics of the measured accuracy values, a linear regression line of accuracy, depending on CT dose, was constructed to illustrate a potential influence of low CT dose on low accuracy. For inferential statistical analysis of the dose dependence of the accuracy, a nonparametric Wilcoxon signed rank test with a Bonferroni correction for multiple comparison was used.

\section{Endoscopy with Low-Dose CAS-CT}

CT scans of a cadaver head were obtained with low-dose scanning. Computer-assisted endoscopy was performed by 5 ENT surgeons ex- 

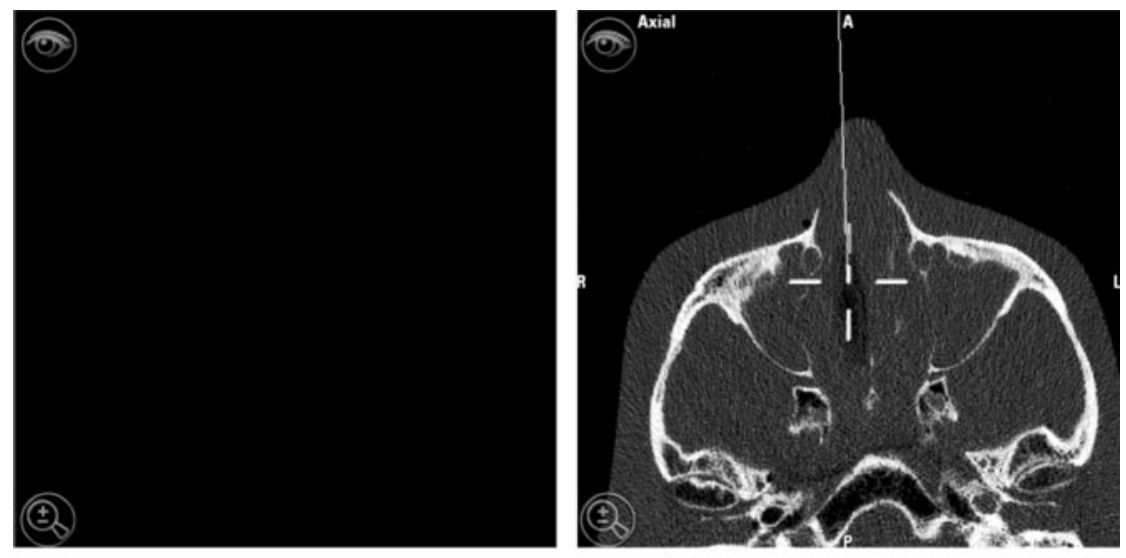

Fig 2. Screenshot from the navigation system display shows the information the ENT surgeon has available during the intervention. A low-dose dataset (CTDI vol of $3.1 \mathrm{mGy}$ ) was used.
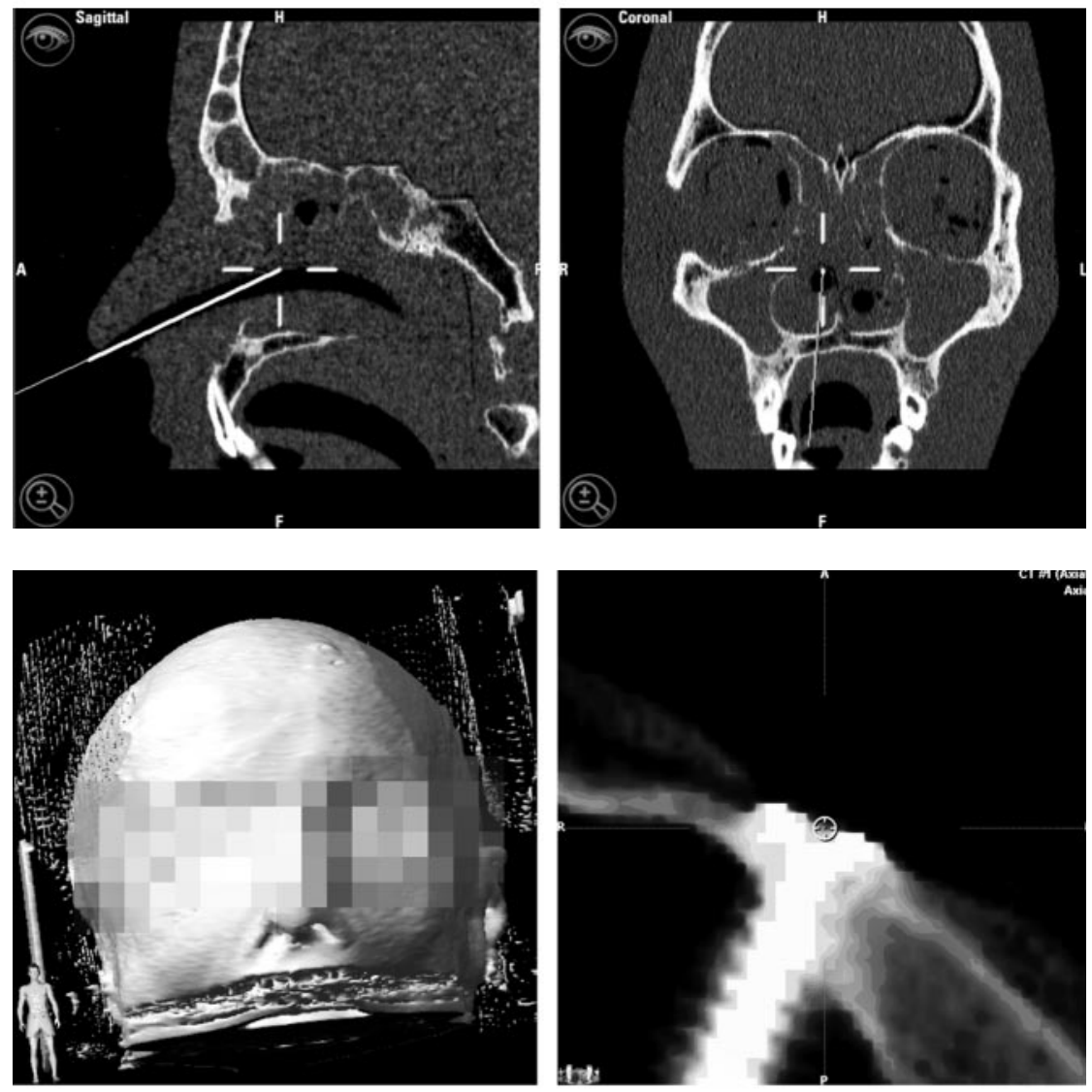

Fig 3. This screenshot from the planning software iPlan shows a CT dataset with coronal and sagittal reformations. The central screw head pit of the screw in the zygomatic bone is heavily magnified for positioning the marker circle used for the accuracy measurements.
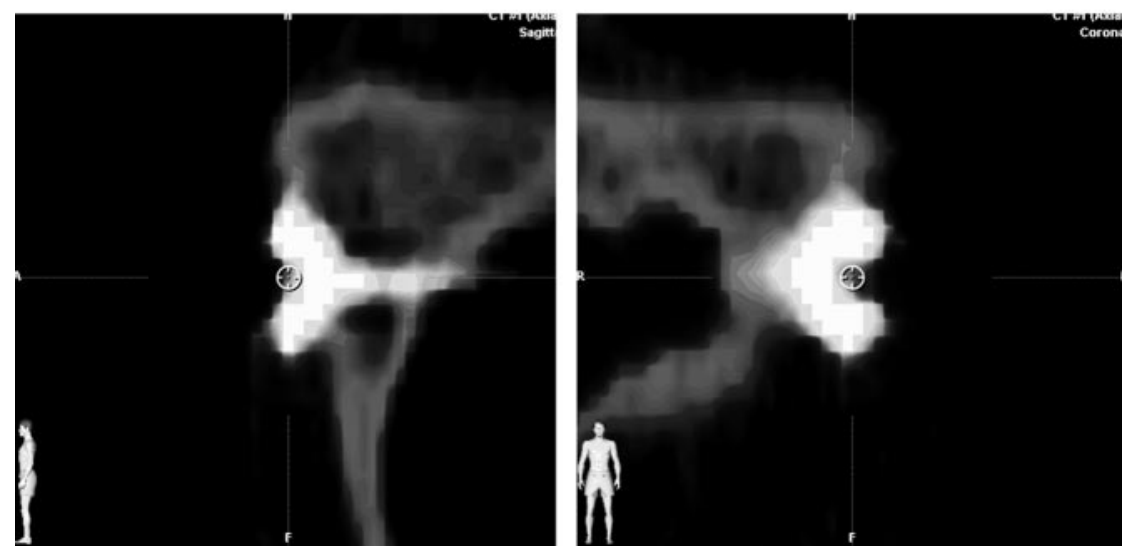

perienced from 1 to 12 years (median, 3 years). Six anatomic locations (carotid canal, optic canal, middle concha attachment, uncinate process, ethmoid bulla, and anterior wall of sphenoid sinus) had to be identified on both sides. Anatomic locations to be assessed were chosen according to their relevance to the surgeon. For example, not the optic nerve (which is not well delineated on edge-enhanced CT-im- 


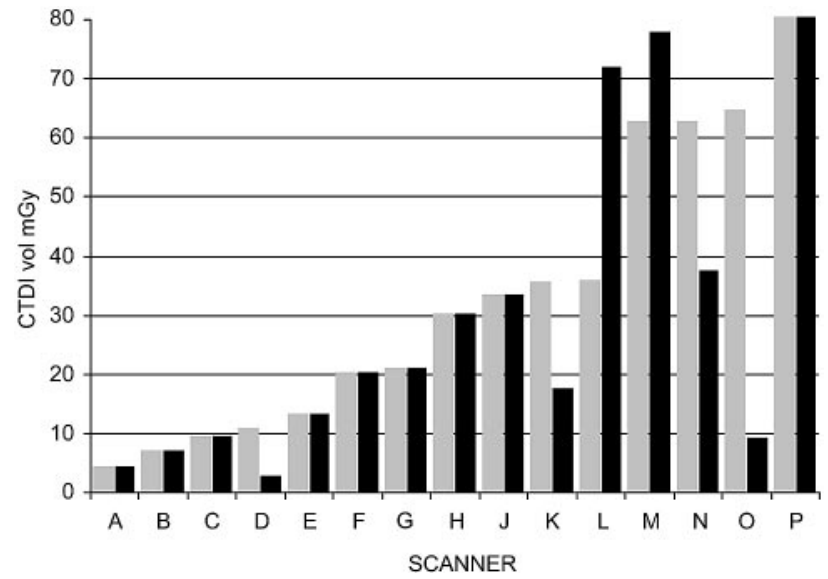

Fig 4. Results of the dose survey. Doses used for CAS-CT (gray) and diagnostic sinus CT (black) in 15 CT scanners. 0 is our institution before the CAS-CT protocol was adapted.

ages) but the bony borders defining the dangerous space around the optic nerve should be discernible. In addition, overall image quality had to be judged on a scale from 1 to 10 , considering the question "Would you be able to perform endoscopy on a real patient with these images?" One week before the experiment, participants were informed about the study background and low-dose scanning was demonstrated. During the following week, opportunities were given repeatedly for becoming acquainted with low-dose image quality. During the experiment, participants were blinded to the doses.

\section{Results}

\section{Dose Survey}

Nineteen of 30 questionnaires were returned (response rate, 63\%), 5 institutions did not perform CAS-CT, and 14 institutions reported data for 15 scanners. Scanners reported included 10 different models from 4 manufacturers; all scanners were multisection technology (2 section to 64 section). $\mathrm{CTDI}_{\mathrm{vol}}$ for CAS-CT and sinusitis CT is shown in Fig 4. Variation between minimal and maximal doses used for CAS-CT was 18 -fold. In 9 of 15 scanners, the same dose was used for CAS-CT and for sinusitis CT; the dose for CAS-CT was higher in 4 scanners and lower in 2 scanners. Five of 14 institutions used an image-guidance system from the same manufacturer as ours. Other systems used included Stryker Leibinger (Freiburg, Germany), Pole Star (Medtronic, Minneapolis, Minn), and Instatrak (GE Healthcare, Milwaukee, Wis).

\section{Evaluation of Registration Feasibility and Dose Dependence of Accuracy}

Phantom surface registration was readily possible with all doses. There was no significant difference in accuracy between the different low-dose scans (Fig 5), and there was no significant difference between the highest dose used as a reference and the low-dose scans $(P>.05$, Wilcoxon signed rank, Bonferroni-corrected for multiple testing). For the highest dose (CTDI $_{\mathrm{vol}}$ of $65 \mathrm{mGy}$ ), mean accuracy was $1.20 \mathrm{~mm}$ (SD, 0.77); and for the lowest dose $\left(\mathrm{CTDI}_{\mathrm{vol}}\right.$ of $\left.1.1 \mathrm{mGy}\right)$, the mean accuracy was $1.40 \mathrm{~mm}(\mathrm{SD}, 1.04)$.

\section{Endoscopy with Low-Dose CAS-CT}

In general, identification of the anatomic reference points was also readily possible at very low doses. At $3.1 \mathrm{mGy}$, all ana-

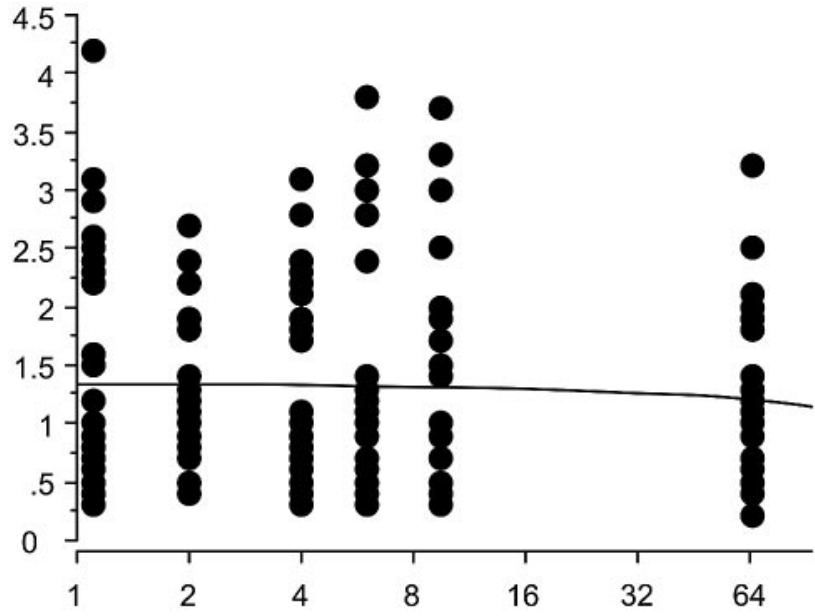

Fig 5. Scatterplot of accuracy measurements in millimeters for screw-marked points depicted on the $y$-axis. CAS-CT dose used in $\mathrm{mGy}$ depicted on the logarithmic $x$-axis. Each column represents the accuracy in $\mathrm{mm}$ for 7 screw-marked points on 4 cadaveric heads for registration with CAS-CT at a given dose. The regression line shows no clinically significant slope for accuracy with exponential growth of the radiation dose in the dose interval tested. Note that slight irregularities of the regression line due to the limited resolution of the software used for statistical analysis were straightened by using Photoshop (Adobe Systems, San Jose, Calif), without altering the content of the graphic.

tomic landmarks were identified confidently. At $1.1 \mathrm{mGy}$, the right internal carotid artery canal was judged not to be identifiable by all surgeons, and at $2 \mathrm{mGy}, 1$ surgeon judged the right internal carotid canal borders not to be readily identifiable. All other reference points were identified readily, even at the lowest dose. Image quality was assigned a mean value of 9.4 on a 10 -point scale for the highest dose and 8.0 for the lowest dose.

\section{Discussion}

Low-dose protocols for sinus CT allow sufficient visualization of structures with high intrinsic contrast, such as bony septa or air-fluid levels, and are generally used to confirm or rule out acute or chronic sinusitis but may not warrant enough softtissue contrast for delineation of a tumor or an abscess. These protocols are well established for indications in which limited soft-tissue contrast is acceptable, ${ }^{9-11}$ but no systematic evaluation of the feasibility of low-dose CT for computer-assisted navigation in FESS has been performed until now. Husstedt et $\mathrm{al}^{13}$ found a sufficient navigation accuracy for a low-dose protocol by using $140 \mathrm{kV}$ and $40 \mathrm{~mA}$ with a single-section scanner. These dose parameters result in a dose, however, that is above that which should be considered a low-dose protocol in modern multisection scanners. Due to the practical need to reduce CT-associated radiation exposure to our patients, we decided to investigate currently used doses for CAS-CT and technically determined lower dose limits for computer-assisted sinus navigation.

Our dose survey shows a vast variation of doses used by different institutions for CAS-CT, with the highest dose being 18 times higher than the lowest. This difference might be partially justified by technical differences between scanners because discrepancies in tube and detector design, filtration, and gantry geometry have an influence on the dose and because the same CTDI does not result in the same image quality for all scanners. ${ }^{14}$ All scanners involved were modern multisection technology, though, and the difference cannot be explained by 

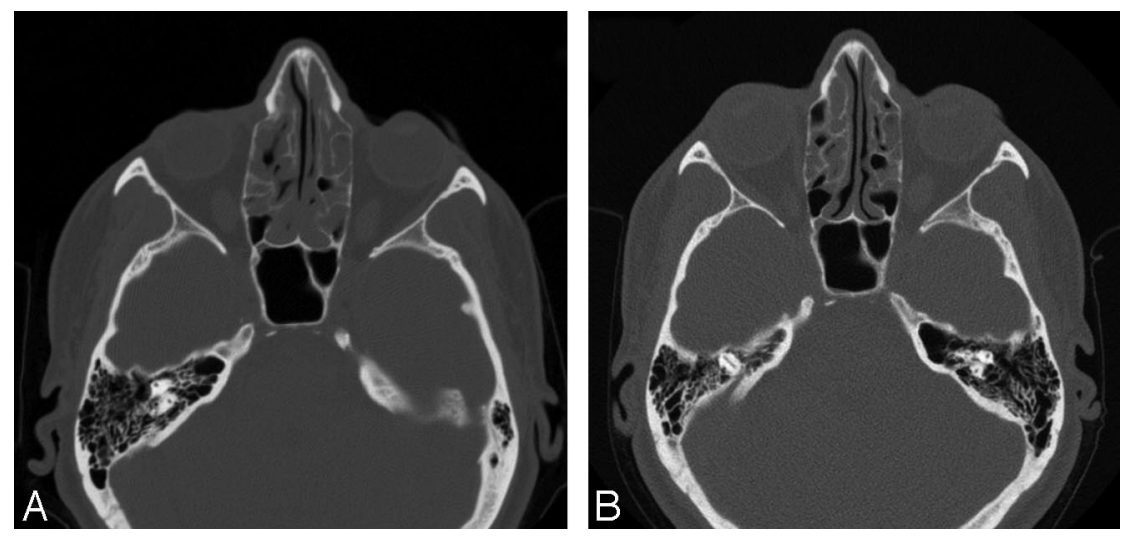

Fig 6. Sinus CT in an edge-enhanced (bone) algorithm performed with a CTDI $\mathrm{vol}_{\mathrm{v}}$ of $65 \mathrm{mGy}(A)$ and with a CTDI $\mathrm{vol}_{\mathrm{v}}$ of 9.5 $\mathrm{mGy}(B)$. The sevenfold dose difference is not appreciated well, though image $B$ is obviously noisier than $A$.

technical factors alone. This opinion was supported by Koller et $\mathrm{al},{ }^{15}$ who found a sevenfold CTDI $_{\mathrm{vol}}$ difference for diagnostic sinus CT between institutions, all of which used the same scanner model.

Because for sinusitis, only structures with a high intrinsic contrast need to be visualized, sinus CT is particularly suitable for low-dose scanning. On the other hand, such a limited approach might fail to show alternative diagnoses like an intracranial tumor clinically imitating a sinusitis ("frontal headache"). There is a controversy among radiologists about lowdose sinus scanning, and some radiologists reject low-dose scanning, preferring full tissue contrast for every examination in order to avoid missing alternative findings. ${ }^{16}$ In our eyes, the vast dose variation between different institutions mainly reflects this controversy.

Nevertheless, the results of our dose survey allow the conclusion that there is no technical factor justifying a higher radiation dose for CAS-CT than for sinusitis CT. Most institutions perform both examinations with the same parameters, some with a dedicated low-dose protocol; and those that use different doses do not show evidence of a uniform trend toward using a higher dose for CAS-CT.

Because we found that low-dose computer-assisted navigation is feasible and already being performed by other institutions, we modified our CAS-CT protocol stepwise in collaboration between neuroradiologic and ENT departments until the dose used for sinusitis CT was reached. We apply this protocol now to those patients in whom clinically an uncomplicated inflammatory mucosal affection (sinusitis or polyposis) is probable. The image-quality degradation on lowering from 210 to $30 \mathrm{mAs}$ is not well perceived on the edge-enhanced images used for navigation (Fig 6) and is tolerated well by the ENT surgeons. To smooth the surfaces produced by the surface-rendering algorithm, we adapted thresholds once during the dose-adaptation process. No problems were observed during patient registration or interventions afterward.

In the second experimental part of our study, minimal doses required for registration and safe navigation were investigated in experimental models. Registration proved to be feasible, irrespective of radiation dose, and for navigation accuracy, no statistically significant dose-dependence was observed. The accuracy values we found for our VectorVision tool are comparable with others cited in the literature. ${ }^{1,3}$ Therefore, technical accuracy does not need to be considered as a factor limiting dose reduction.
Our experiment testing surgeon performance with lowdose scanning showed that image quality is not very critical either because ENT surgeons were able to identify anatomic structures with doses as low as approximately a third of our diagnostic low-dose protocol. Surgeons do not primarily use the CT scanning for orientation but work with the endoscope, and CT images are used more to verify the position of the instruments within anatomic compartments. ${ }^{3}$ They, therefore, only need a basic image quality. Besides, images calculated with an edge-enhancing algorithm are used for navigation, and in these images, soft-tissue details are not easily discernible anyway. In practice though, dose reduction is limited by the fact that CAS-CT usually serves diagnostic purposes as well and that conspicuousness of anatomic details, beyond those details the surgeons needs to identify, should be warranted for the radiologist.

Therefore, we do not advocate the use of extremely lowdose CT for navigation in FESS on a routine basis. However, the findings of our study point out 2 relevant points: Many patients undergo CAS-CT at much higher doses than necessary, and dose reduction is far less critical than might be expected. We consider the results of the present study of particular interest in light of the growing awareness of potential hazards from CT-associated radiation exposure in the public and among radiologists. Neuroradiologists are increasingly called on to reduce radiation exposure from $\mathrm{CT}^{17,18}$; and in this context, questions concerning the feasibility of dose reduction in CAS-CT will arise.

In practice, in regard to performing CAS-CT, we recommend checking the currently used dose parameters and carrying out, where appropriate, a pragmatic dose reduction down to a level typically used for low-dose diagnostic scans, such as those used for patients treated for rhinosinusitis. For modern multisection scanners, generally $120 \mathrm{kV}$ and 20-50 mAs will suffice. ${ }^{11,19}$ A recent publication ${ }^{20}$ showed that significant dose reduction can result from the use of dose-modulation techniques in neuroradiologic CT. Using dose modulation also for CAS-CT is, therefore, recommended. In this case, an upper limit for the tube current must be set to avoid excessive tube currents induced by metallic dental implants.

Low-dose scanning should not be used, of course, when soft-tissue contrast is an issue, as in the case of tumor surgery. At our institution, approximately 100 sinus surgery procedures are performed annually with computer-assisted naviga- 
tion, 90 of these for chronic rhinosinusitis; hence, most patients benefit from the new dose-reduced protocol.

When a recent diagnostic scan is available and a dataset must be acquired only for navigation purposes and/or when radiation protection is a major concern (as for pregnant women or children), even lower doses (about a third of a typical diagnostic dose level) may be considered. Moreover, we scan all patients for whom FESS might be necessary in the future a priori with a wide FOV (including the tip of the nose), allowing the dataset to be used for navigation later, thus avoiding excessive radiation exposure from repeat scanning.

Apart from the stochastic risk resulting from radiation exposure, there is 1 potential deterministic risk associated with radiation in sinus CT: That is the risk of cataract development. A recent study ${ }^{6}$ found lens doses in navigation CT for pituitary surgery far below the threshold inducing cataracts in humans. As for intracranial navigation, a far better tissue contrast is required and, therefore, considerably higher radiation doses are applied than for sinus navigation; the lens dose in a single sinus CT is negligible.

Our study has some limitations. Different scanner types may need significantly higher or lower doses for the same image quality; hence, dose-relevant parameters mentioned in our study cannot be transferred directly to other scanners. That is why we give our recommendation in a rather generic way (use a dose level as typically used in low-dose diagnostic sinusitis scans, and generally $120 \mathrm{kV}$ and 20-50 mAs will suffice). Other navigation systems (especially if they should use a significantly different image processing and registration method) may be more susceptible to image-quality degradation induced by low radiation dose.

The significance of the findings from our experimental low-dose endoscopy is somewhat limited by the fact that endoscopy in a cadaver head, where no mucosal swelling and bleeding occur, is easier than in a real patient and that the recognition of bony lamellae in a diseased patient may be hampered by chronic inflammation. As a consequence of these limitations, we recommend proceeding stepwise and in close collaboration with the ENT surgeon to find an acceptable image quality when undertaking dose reduction.

Last, our study is only an experimental trial under laboratory conditions, and the clinically most relevant issue (the number of complications) was not investigated. Evaluation of the noninferiority of low-dose CAS-CT in the clinical routine would have to be investigated in a clinical trial, which needs to include a large number of patients because of the low frequency of intraoperative complications.

\section{Conclusions}

In this study, the first radiation-dose survey for CT used for computer-assisted navigation in FESS is presented, and minimal radiation doses required are investigated experimentally. A wide dose range is used among radiology departments, reflecting varying attitudes toward diagnostic needs and concern about radiation exposure among radiologists.

The only practically relevant limit for dose reduction for CAS-CT is imposed by the ENT surgeon's need for a basic image quality and the radiologist's need for diagnostic image quality, whereas the navigation system tested does not impose technical limits for dose reduction. The radiation doses for CAS-CT can be reduced far below those currently applied by most institutions. We recommend using those doses that are commonly applied in typical low-dose diagnostic sinus CT. When no diagnostic image quality is required, dose reduction down to approximately a third of a typical diagnostic low-dose sinus CT can be considered.

\section{Acknowledgments}

We thank fellow ENT surgeons Claudia Candreia, Matthias Hagemann, and Andreas Arnold for their collaboration; Gudrun Herrmann, Institute of Anatomy, University of Berne, for providing the anatomic specimens; Martin Kompis, Department of Clinical Research, University of Berne, for methodologic advice; and Eva Clamann for correcting the manuscript.

\section{References}

1. Stelter K, Andratschke M, Leunig A, et al. Computer-assisted surgery of the paranasal sinuses: technical and clinical experience with 368 patients, using the Vector Vision Compact system. J Laryngol Otol 2006;120:1026-32. Epub 2006 Sep 25

2. Anon JB, Klimek K, Mösges R, et al. Computer-assisted endoscopic sinus surgery: an international review. Otolaryngol Clin North Am 1997;30:389-401

3. Metson R, Cosenza M, Glicklich RE, et al. The role of image-guidance systems for head and neck surgery. Arch Otolaryngol Head Neck Surg 1999;125:1100-04

4. Heermann R, Schwab B, Issing PR, et al. Navigation with the StealthStation in skull base surgery: an otolaryngological perspective. Skull Base 2001; 11:277-85

5. Mann W, Klimek L. Indications for computer-assisted surgery in otorhinolaryngology. Comput Aided Surg 1998;3:202-04

6. Ulmer S, Schulz E, Moeller B. Radiation dose of the lens in trans-sphenoidal pituitary surgery: pros and cons of a conventional setup using fluoroscopic guidance and CT-based neuronavigation. AJNR Am J Neuroradiol 2007; 28:1559-64

7. Hsu L, Fried MP, Jolesz FA. MR-guided endoscopic sinus surgery. AJNR Am J Neuroradiol 1998;19:1235-40

8. Brenner DJ, Elliston CD, Hall EJ, et al. Estimated risks of radiation-induced fatal cancer from pediatric CT. AJR Am J Roentgenol 2001;176:289-96

9. Marmolya G, Wiesen EJ, Yagan R, et al. Paranasal sinuses: low-dose CT. Radiology 1991;181:689-91

10. Sohaib SA, Peppercorn PD, Horrocks JA, et al. The effect of decreasing $\mathbf{m A s}$ on image quality and patient dose in sinus CT. Br J Radiol 2001;74:157-61

11. Tack D, Widelec J, De Maertelaer V, et al. Comparison between low-dose and standard-dose multidetector CT in patients with suspected chronic sinusitis. AJR Am J Roentgenol 2003;18:939-44

12. Caversaccio M, Langlotz F, Nolte LP, et al. Impact of a self-developed planning and self-constructed navigation system on skull base surgery: 10 years' experience. Acta Otolaryngol 2007;127:403-07

13. Husstedt H, Heermann R, Becker H. Contribution of low-dose CT-scan protocols to the total positioning error in computer-assisted surgery. Comput Aided Surg 1999;4:275-80

14. Lewis M. Radiation dose issues in multi-slice CT scanning. ImPACT technology: update no 3-MHRA. 2005. Available at: www.impactscan.org/ download/msctdose.pdf. Accessed November 1, 2008

15. Koller CJ, Eatough JP, Bettridge A. Variations in radiation dose between the same model of multislice CT scanner at different hospitals. $\mathrm{Br} J$ Radiol 2003;76:798-802

16. Hudgins PA, Mukundan S. Screening sinus CT: a good idea gone bad? AJNR Am J Neuroradiol 1997;18:1850-54

17. Smith AB, Dillon WP, Gould R, et al. Radiation dose-reduction strategies for neuroradiology protocols. AJNR Am J Neuroradiol 2007;28:1628-32

18. Fox AJ. Use of the lowest necessary radiation dose. AJNR Am J Neuroradiol 2004;25:519

19. Mulkens T, Salgado R, Bellinck P. Dose optimization and reduction in CT of the head and neck, including brain. In: Tack D, Gevenois A, eds. Dose Reduction in Multi-Detector Computed Tomography. Berlin, Germany: Springer-Verlag; 2007:136-51

20. Smith AB, Dillon WP, Lau BC, et al. Radiation dose reduction strategy for CT protocols: successful implementation in neuroradiology section. Radiology 2008;247:499-506. Epub 2008 Mar 27 